BBA 66260

\title{
EFFECT OF ANIONS ON THE CATALYTIC ACTIVITY OF PIG LIVER GLYCOLIC ACID OXIDASE*
}

MARILYN SCHUMAN"* AND VINCENT MASSEY

Department of Biological Chemistry, The University of Michigan, Ann Avbor, Mich. 48104 (U.S.A.)

(Received September 8th, 1970)

SUMMARY

I. Pig liver glycolic acid oxidase (glycolate: $\mathrm{O}_{2}$ oxidoreductase, EC I.I.3.I) can function with either 2,6-dichlorophenolindophenol (DCIP) $\left(v_{\max }=\right.$ I250 moles of glycolate per mole of flavin per min) or $\mathrm{O}_{2}\left(v_{\max }=620\right.$ moles of glycolate per mole of flavin per min $)^{9,10}$ as the electron acceptor.

2. Straight-chain monocarboxylic acids are non-competitive inhibitors of this enzyme with either glycolate or glyoxalate as the variable substrate and DCIP as the electron acceptor. Dicarboxylic acids are competitive inhibitors when glycolate is the variable substrate but they are non-competitive inhibitors when glyoxalate is the variable substrate. Phosphate and arsenate cause enzyme activation.

3. The binding affinity of the enzyme for monocarboxylic acids is proportional to the number of carbon atoms in the alkyl residue $(n)$, and a straight line is obtained when $\log K_{i}$ is plotted versus $n$. These results suggest that electrostatic and hydrophobic forces, associated with a positive charge and a hydrophobic region at the active site, contribute to the binding affinity of monocarboxylic acids.

4. The binding affinity of the enzyme for oxalate is surprisingly high when compared with monocarboxylic acids. A decreased binding affinity is observed for other dicarboxylic acids as the number of carbon atoms increases. These results suggest that the complex of the enzyme with oxalate involves electrostatic interaction of both carboxylate groups of oxalate with two adjacent positively charged groups at the active site. A similar bidentate complex may explain the binding affinity of the enzyme for $\alpha$-hydroxy acid substrates.

5. Conditions under which non-competitive inhibition can approximate competitive inhibition are discussed to account for differences in the inhibition patterns observed when glycolate and glyoxalate are used as the variable substrate.

\footnotetext{
Abbreviations: DCIP, 2,6-dichlorophenolindophenol; Hepes, $N$-2-hydroxyethylpiperazine$N^{\prime}$-2-ethanesulfonic acid.

* The data in this paper are taken from a dissertation presented by M. S. to the Faculty of the Graduate School of The University of Michigan in partial fulfillment of the requirements for the degree of Doctor of Philosophy.

${ }^{*}$ Present address: Fachbereich Biologie, Universität Konstanz, Konstanz, Germany.
} 


\section{INTRODUCTION}

Studies on the effects of anions on the visible absorption spectrum of glycolic acid oxidase (glycolate: $\mathrm{O}_{2}$ oxidoreductase, EC I.I.3.I.) have indicated that there is at least one positively charged amino acid and also a hydrophobic region of the protein near the flavin prosthetic group ${ }^{1}$. Anions which cause spectral perturbations in other flavoproteins also act as enzyme inhibitors ${ }^{2-6}$. Studies on yeast lactic acid dehydrogenase ${ }^{7}$ and the cholinesterases ${ }^{8}$ indicate that the inhibition constants of an homologous series of inhibitors can yield important information about the active surface of an enzyme. The present communication describes the inhibition of glycolic acid oxidase by homologous series of monocarboxylic and dicarboxylic acids. These studies indicate that the positively charged groups function in the binding of $u^{-}$ hydroxy acid substrates. A model is proposed for the enzyme-substrate complex and discussed in terms of the observed properties of the active site. Conditions under which non-competitive inhibition can approximate competitive inhibition are also discussed to account for differences in the inhibition patterns observed when different hydroxy acids are used as substrates.

\section{METHODS AND MATERIALS}

Glycolic acid oxidase was purified from pig liver by a modification ${ }^{1}$ of the method of Dickinson". Inhibition by monocarboxylic and dicarboxylic acids was studied using DCIP as the electron acceptor. Unless otherwise stated, enzyme activity was determined as previously described ${ }^{1}$ except that the concentration of potassium phosphate buffer (approx. o.I M) was varied to maintain a constant total salt concentration (substrate, inhibitor plus phosphate) of $0.174 \mathrm{M}$.

It was found that after pipetting many aliquots from a dilute sample of enzyme, enzyme activity decreased. A control sample of enzyme left undisturbed for the same period did not lose activity. The reason for these losses was not discovered. Each set of kinetic experiments described in this paper was obtained with enzyme freshly diluted from a stock solution into O.I M sodium phosphate $(\mathrm{pH} 7.0)$ containing bovine serum albumin $(5 \mathrm{mg} / \mathrm{ml})$ to enhance stability. The diluted sample of enzyme was divided into several aliquots, and each aliquot used for no more than ten assays. Each assay was done in duplicate; the average of these two assays was used for subsequent kinetic analysis.

With the exceptions noted below, all reagents were obtained as previously described ${ }^{1}$. Propionic, valeric, hexanoic, malonic and succinic acids were purchased from Eastman Organic Chemicals. Glacial acetic acid was obtained from Baker Chemical Company. Butyric acid was from F. P. Jay Chemicals. $N$-2-Hydroxyethylpiperazine- $N^{\prime}$-2-ethanesulfonic acid (Hepes) was purchased from Calbiochem. Potassium arsenate was obtained from Matheson, Coleman and Bell. The monocarboxylic acids, except acetic acid, were distilled before use to remove colored impurities. All other reagents were used without further purification. Substrates and inhibitors were neutralized with $\mathrm{NaOH}$ before use. 
RESULTS

\section{Kinetics of the glycolate-DCIP reaction}

Fig. I shows a plot of reciprocal turnover number versus reciprocal glycolate concentration at different concentrations of DCIP. The lines at different concentrations of DCIP are convergent. Similar results are obtained when the fixed and variable substrates are reversed. At glycolate concentrations higher than $1.7 \cdot 10^{-3} \mathrm{M}$

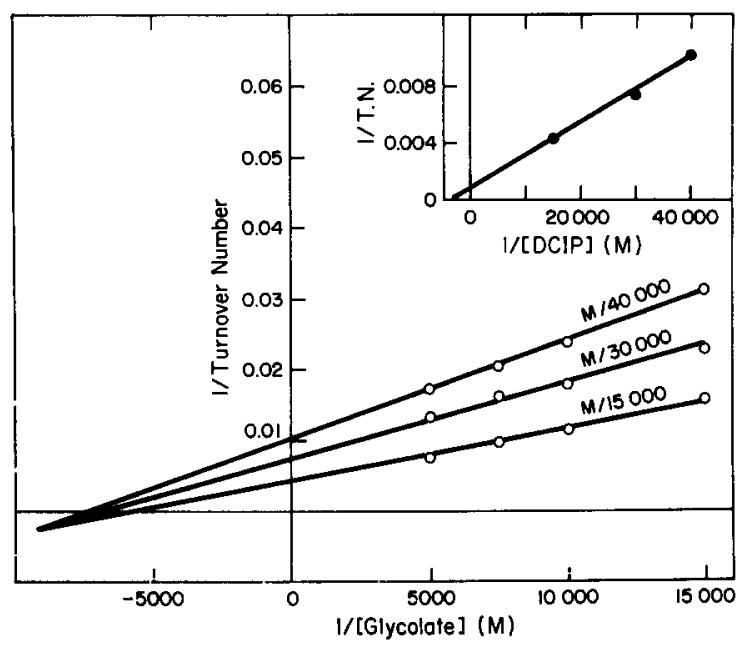

Fig. 1. Plot of reciprocal turnover number versus reciprocal glycolate concentration. The concentrations given above the lines refer to the concentrations of DCIP used. Turnover number is defined as moles of glycolate oxidized per min per mole of enzyme-flavin. Flavin is estimated by its measured absorbance at $45^{\circ} \mathrm{m} \mu$. The inset shows a plot of reciprocal turnover number at infinite glycolate concentration versus reciprocal DCIP concentration.

excess substrate inhibition is observed. The intercepts of the double reciprocal plots were plotted versus reciprocal substrate concentration (e.g. see inset of Fig. I) to obtain values for $v_{\max }$ (1250 moles of glycolate per mole of flavin per min) and $K_{m}$ $\left(K_{m}\right.$ (glycolate $\left.)=4.2 \cdot 10^{-4} \mathrm{M}, K_{m}(\mathrm{DCIP})=2.8 \cdot 10^{-4} \mathrm{M}\right)$. A $v_{\max }$ of 620 moles of glycolate per mole of flavin per min was obtained for the glycolate: $\mathrm{O}_{2}$ reaction ${ }^{9,10}$. Although the two sets of data are not strictly comparable, it is clear that the enzyme can function with $\mathrm{O}_{2}$ or DCIP as electron acceptor.

\section{Inhibition by monocarboxylic acids}

The inhibition of glycolate oxidation at several different concentrations of acetate is shown in Fig. 2. The inhibition is non-competitive with respect to glycolate since both the slopes and intercepts of the Lineweaver-Burk plot are affected by the inhibitor.

Eqn. I represents linear non-competitive inhibition.

$$
\frac{\mathbf{I}}{v}=\frac{K_{\mathrm{A}}}{v_{\max }}\left[\mathbf{I}+\frac{[I]}{K_{i \text { (slope) }}}\right] \frac{\mathbf{I}}{[\mathrm{A}]}+\frac{\mathbf{I}}{v_{\max }}\left[\mathbf{I}+\frac{[I]}{K_{i \text { (intercept) }}}\right]
$$




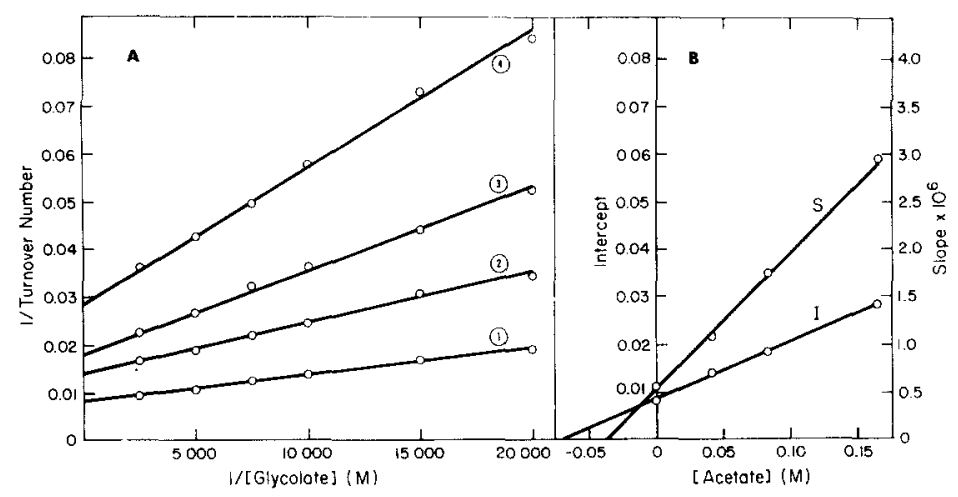

lig. 2. Inhibition of glycolate oxidation by acetate. A. Plot of reciprocal turnover number versus reciprocal glycolate concentration. I,ine 1 , no inhibitor; Line $2,4.17 \cdot 10^{-2} \mathrm{M}$ acetate; Line 3 , $8.34 \cdot 10^{-2} \mathrm{M}$ acetate; Line $4.1 .67 \cdot 10^{-1} \mathrm{M}$ acetate. B. Plot of intercepts (I) and slopes (S) versus acetate concentration.

The values for $K_{i \text { (slope) }}$ and $K_{i \text { (intercept) }}$ represent those concentrations of inhibitor that double the uninhibited slope and intercept, respectively. The values for $K_{i}$ are easily obtained by replotting slopes and intercepts versus the inhibitor concentration according to the method outlined by (LFLANI) ${ }^{11}$. The intercepts of the slope and intercept plots on the horizontal axis give the $K_{i \text { (slope) }}$ and $K_{i \text { (intercept), respectively. }}$ Values of $3.7 \cdot \mathrm{IO}^{-2}$ and $7 . \mathrm{I} \cdot \mathrm{IO}^{-2} \mathrm{M}$ are obtained for $K_{i \text { (slope) }}$ and $K_{i \text { (intercept), re- }}$ spectively, from the secondary plot of the data obtained with acetate (Part $B$ of Fig. 2). Similar non-competitive inhibition of glycolate oxidation is observed with other members of the homologous series of straight-chain monocarboxylic acids. The pattern of inhibition is unchanged when glyoxalate is used instead of glycolate as the primary substrate. The inhibition constants obtained for the monocarboxylic acids are summarized in Table I.

It is seen that the values for $K_{i \text { (slope) }}$ are always smaller than the corresponding

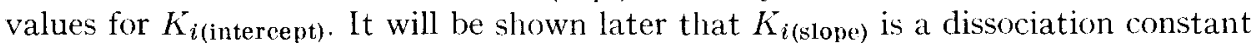
for the complex of inhibitor with the oxidized enzyme, while $K_{i \text { (intercept) }}$ is an apparent dissociation constant for the complex of inhibitor with a catalytic intermediate of the enzyme. The substrate used should not affect $K_{i \text { (slope) }}$ but may affect $K_{i \text { (intercept) }}$.

TABI, I T

MONOCARBOXYLIC ACID INHIBITION CONSTANTS

Inhibition constants are molar inhibition constants.

\begin{tabular}{|c|c|c|c|c|}
\hline Inhibitor & $\begin{array}{l}\text { Glycolate } \\
K_{\text {irslopes }} \\
(M)\end{array}$ & $\begin{array}{l}\text { Glycolate } \\
\text { Ki(intercept) } \\
(M)\end{array}$ & $\begin{array}{l}\text { Glyoxalate } \\
K_{\text {ifstope }} \\
(M)\end{array}$ & $\begin{array}{l}\text { Glyoxalate } \\
K_{\text {ifintercept }} \\
(M)\end{array}$ \\
\hline Acetate & $3.7 \cdot 10^{-2}$ & $7.1 \cdot 10^{-2}$ & $4.8 \cdot 10^{-2}$ & $2.3 \cdot 10^{-11}$ \\
\hline Propionate & $9 \cdot 5 \cdot 10^{\cdots 3}$ & $2.0 \cdot 10^{-2}$ & $1.7 \cdot 10^{-2}$ & $6.8 \cdot 10^{-2}$ \\
\hline Butyrate & $3.6 \cdot \mathrm{IO}^{-3}$ & $7 \cdot 3 \cdot 10^{-3}$ & & \\
\hline Valerate & $\mathrm{T} \cdot 3 \cdot 10^{-3}$ & $2.3 \cdot 10^{3}$ & $1.2 \cdot 10^{-3}$ & $3.8 \cdot 10^{-3}$ \\
\hline Hexanoate & $3.0 \cdot 10^{4}$ & $1.1 \cdot 10^{-3}$ & & \\
\hline Heptanoate & $9.2 \cdot 10^{--5}$ & $4.7 \cdot 10^{-1}$ & & \\
\hline
\end{tabular}

BiochiA. Biophys. Acta, 227 (1971) 521-5.37 
Table I shows that $K_{i \text { (slope) }}$ and $K_{i \text { (intercept) }}$ are both independent of the substrate used when glycolic acid oxidase is inhibited by monocarboxylic acids.

Both $K_{i \text { (slope) }}$ and $K_{i \text { (intercept) }}$ decrease as the number of carbon atoms in the alkyl side chain increases (Table I). It is quite probable that one component of the binding energy derives from electrostatic interaction of the negative carboxylate group of the inhibitor molecule with a positively charged group near the active site of the enzyme. However, the alkyl residue must also contribute to the binding affinity since the inhibition constants become progressively smaller as the alkyl residue becomes larger. If the structure of the active site imposes no steric restrictions on the paraffin chains, it is to be expected that each carbon atom will contribute equally to the binding affinity. Under these conditions, the free energy change associated with the binding of a monocarboxylic acid containing $n$ carbon atoms in its alkyl residue can be represented by Eqn. 2 .

$$
\Delta F_{\text {Net }}=\Delta F_{\text {Electrostatic }}+n \Delta F_{-\mathrm{CH}_{2}-}
$$

Inhibition constants are dissociation constants (or proportional to dissociation constants) ${ }^{11}$. Therefore $-R T \ln K_{i}$ can be substituted for $\Delta \mathrm{F}_{\text {Net }}$ (Eqn. 3).

$$
-R T \ln K_{1}=\Delta F_{\text {Electrostatie }}+n \Delta F_{-\mathrm{CH}_{2}-}
$$

Fig. 3 shows a plot of $\log _{10}$ inhibition constant as a function of $n$ (these results were obtained for the monocarboxylic acids with glycolate as substrate).

Since we are plotting dissociation constants, rather than association constants, the free energy changes calculated directly from Fig. 3 have a positive value. If the sign is changed from positive to negative we can consider the free energy changes associated with the binding of an inhibitor molecule, rather than its dissociation from the enzyme surface.

A value of $-1200 \mathrm{cal} / \mathrm{mole}$ is obtained for $\Delta F_{\text {Electrostatic }}$ from the intercept at

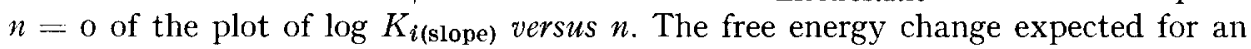

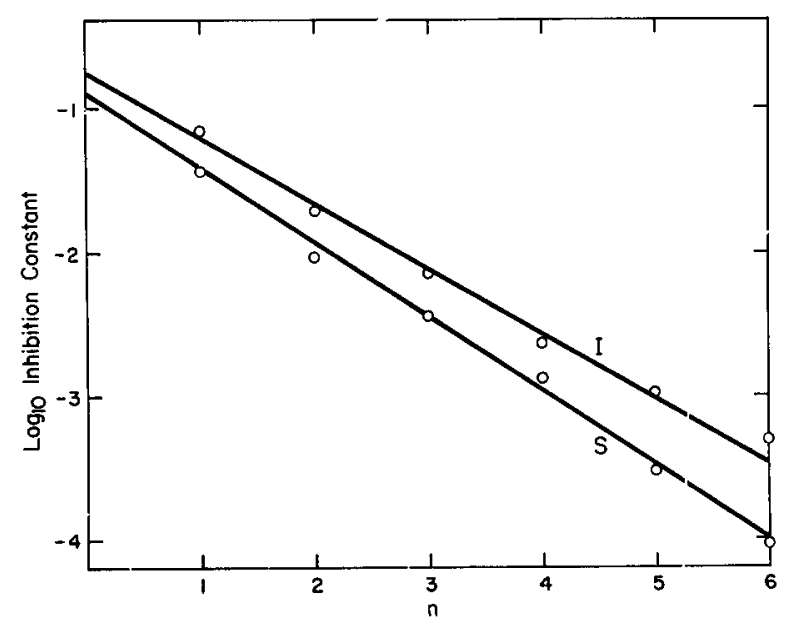

Fig. 3. Plot of $\log _{10}$ monocarboxylic acid inhibition constants as function of the number of carbon atoms $(n)$ in the alkyl residue. Values for $K_{i}$ slope and $K_{i}$ intercept obtained with glycolate as substrate are plotted in Lines $S$ and I, respectively. 
electrostatic interaction can be estimated from the free energy change associated with the formation of monovalent ion pairs in aqueous solution (approx. - I 400 $\mathrm{cal} /$ mole $^{12}{ }^{2}$. Although precise comparison is not possible, the fact that the values are of the same order of magnitude supports the hypothesis that one component of the binding energy of monocarboxylic acids is associated with an electrostatic interaction.

A value for the free energy change for the transfer of one methylene group from solution to the enzyme surface can be calculated from the slope of the plot of $\log K_{i \text { (slope) }}$ versus $n$. The value calculated was $-710 \mathrm{cal} / \mathrm{mole}$. Since the binding affinities of the monocarboxylic acids are inversely proportional to their solubility in water, it is reasonable to assume that this component of the total free energy change arises from a difference in the dielectric constant between the aqueous solvent phase and the protein surface ${ }^{12}$. It is found that the free energy change associated with the transfer of a linear hydrocarbon chain from water to ethanol is $-700 \mathrm{cal} / \mathrm{mole}$ for each methylene group in the chain ${ }^{12}$. This value is similar to the value (- 7 Io cal/

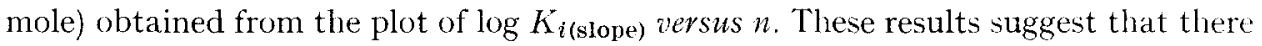
is a region near the active site which is similar to ethanol. This hydrophobic region must be large enough to accommodate an alkyl residue of at least five carbon atoms without steric interference. The inhibition of yeast lactic acid dehydrogenase by monocarboxylic acids follows a pattern similar to that observed with glycolic acid oxidase. Values of 1000 and $500 \mathrm{cal} /$ mole were obtained for $1 \mathrm{~F}$ Electrostatic and $1 F_{-\mathrm{CH}_{2}-}$, respectively ${ }^{7}$.

\section{Inhibition by dicarboxylic acids}

Glycolic acid oxidase is also inhibited by dicarboxylic acids but in this case the inhibition is competitive with respect to glycolate. Part A of Fig. 4 shows the effect of oxalate on the oxidation of glycolate. The inhibition is competitive since only the

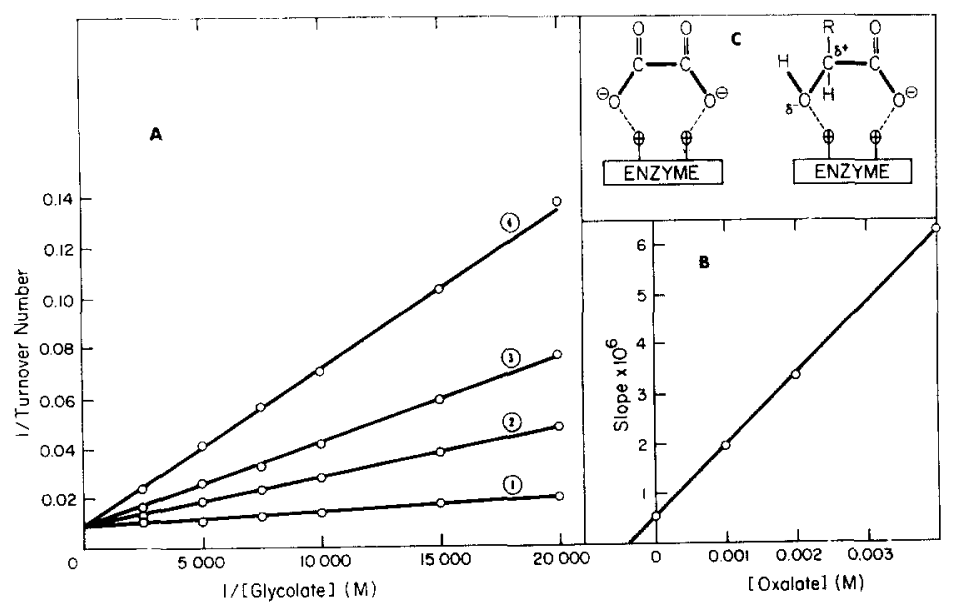

Fig. 4. Inhibition of glycolate oxidation by oxalate. A. Llot of reciprocal turnover number versus reciprocal glycolate concentration. Line 1, no inhibitor; Line $2, \mathbf{1} .0 \cdot 10^{-3} \mathrm{M}$ oxalate: Line 3 .

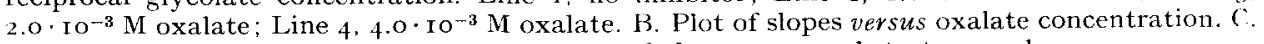
Postulated structures for the enzyme-oxalate and the enzyme-substrate complexes.

Biochim. Biophys. Acta, 227 (1971) $52 \mathrm{I}-537$ 
slope of the Lineweaver-Burk plot is affected by oxalate. Values for the slopes in Part A of Fig. 4 were plotted versus the oxalate concentration (Part B of Fig. 4) and a value for $K_{i \text { (slope) }}$ was determined from the intercept of this secondary plot on the horizontal axis. Values of $3.7 \cdot \mathrm{IO}^{-4}$ and $5.0 \cdot \mathrm{IO}^{-4} \mathrm{M}$ were obtained for $K_{i \text { (slope) }}$ from two different determinations. The affinity of the enzyme for oxalate is surprisingly high compared with its affinity for the monocarboxylic acids. If the binding of oxalate involves electrostatic interaction between only one of its carboxylate groups and a single positive charge at the active site, a binding affinity roughly similar to acetate would be expected. However, the affinity of the enzyme for oxalate is approx. Ioo times greater than that observed for acetate. It is reasonable to assume that the additional forces involved are due to the second carboxylate group of oxalate. The binding of oxalate may involve electrostatic interaction of both carboxylate groups with two adjacent positively charged groups at the active site. Superficially, the $\Delta F_{\text {Net }}$ for oxalate binding might be expected to be approximately twice the value of $\Delta F_{\text {Electrostatic }}$ ( $-\mathrm{I} 200 \mathrm{cal} / \mathrm{mole}$ ) obtained for the monocarboxylic acids, i.e. -2400 $\mathrm{cal} / \mathrm{mole}$. However, since molar equilibrium constants were used to calculate

$A F_{\text {Electrostatic }}$ the value obtained, according to $\mathrm{KAUZMANN}^{13}$ will include a contri-

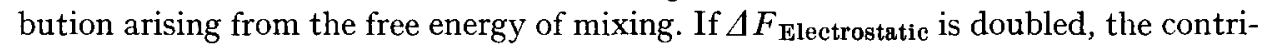
bution from the free energy of mixing is included twice. Therefore, the $\Delta F_{\text {Net }}$ predicted for oxalate binding must be equal to $2\left(\Delta F_{\text {Electrostatic }}\right)-\Delta F_{\text {Mixing. In a system }}$ where two molecules react to form one molecule of product the free energy of mixing at $25^{\circ}$ is $2380 \mathrm{cal} / \mathrm{mole}$. A value of $-4780 \mathrm{cal} / \mathrm{mole}$ is therefore calculated for $A F_{\text {Net }}$. This value corresponds to a dissociation constant of $3.0 \cdot 10^{-4} \mathrm{M}$ which agrees quite well with the observed value of $3.7 \cdot \mathrm{IO}^{-4}-5.0 \cdot \mathrm{IO}^{-4} \mathrm{M}$.

In similarity to results obtained with oxalate, inhibition by malonate and succinate is competitive with respect to glycolate. Unlike the monocarboxylic acids, the inhibition constants for the dicarboxylic acids increase as the number of carbon atoms increases (Table II). This is to be expected if the two positively charged groups are rigidly held at a distance such that the formation of a bidentate enzyme-oxalate complex is sterically favorable. When methylene groups are introduced between the two carboxylate groups the conformation of the acid compatible with such a twopoint electrostatic interaction will be energetically less favorable and the effective concentration of inhibitor molecules will be less than the actual concentration.

In the case of competitive inhibition it is possible to determine the number of inhibitor molecules $(n)$ bound per active site. Eqn. 4 represents linear competitive

TABLE II

DICARBOXYLIC ACID INHIBITION CONSTANTS

Inhibition constants are molar inhibition constants.

\begin{tabular}{|c|c|c|c|}
\hline Inhibitor & $\begin{array}{l}\text { Glycolate } \\
K_{\text {i(slope }} \\
(M)\end{array}$ & $\begin{array}{l}\text { Glyoxalate } \\
K_{\text {i(slope })} \\
(M)\end{array}$ & $\begin{array}{l}\text { Glyoxalate } \\
K_{\text {i(intercept })} \\
(M)\end{array}$ \\
\hline $\begin{array}{l}\text { Oxalate } \\
\text { Malonate } \\
\text { Succinate }\end{array}$ & $\begin{array}{l}4.4 \cdot 10^{-4 \star} \\
9.2 \cdot 10^{-3} \\
1.4 \cdot 10^{-2}\end{array}$ & $\begin{array}{l}4.4 \cdot 10^{-4} \\
8.2 \cdot 10^{-3}\end{array}$ & $\begin{array}{l}1.4 \cdot 10^{-3} \\
2.6 \cdot 1 O^{-2}\end{array}$ \\
\hline
\end{tabular}

* Average of two separate determinations. 
inhibition involving $n$ molecules of inhibitor per active site. Eqn. 5 describes the kinetics in the absence of inhibitor.

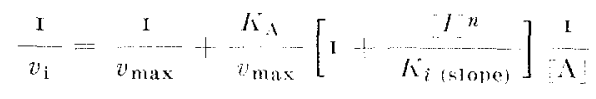

$$
\begin{aligned}
& \frac{\mathrm{I}}{v}=\frac{\mathrm{I}}{v_{\max }}: \frac{K_{\mathrm{A}}}{v_{\max }} \mathrm{A}
\end{aligned}
$$

As indicated by BERGMANX AND SEGAL ${ }^{8}$, suitable transformation of Eqns. 4 and 5 gives Eqn. 6.

$$
\begin{aligned}
& \left.\log \left[\frac{\partial}{v_{i}}-\mathrm{I}\right]=n \log \mid I\right\}+C \\
& \text { where } C=\log \left[\frac{K_{A}}{\left.K_{i \text { (slope })}\left(K_{A}+A_{J}\right]\right)}\right]
\end{aligned}
$$

A plot of $\log \mid\left(\% / \omega_{i}\right)$ - I versus $\log I I$ should give a straight line with a slope equal to $n$. Since the substrate concentration affects only the intercept, plots at different substrate concentrations should give a series of parallel lines. This graphical deter-

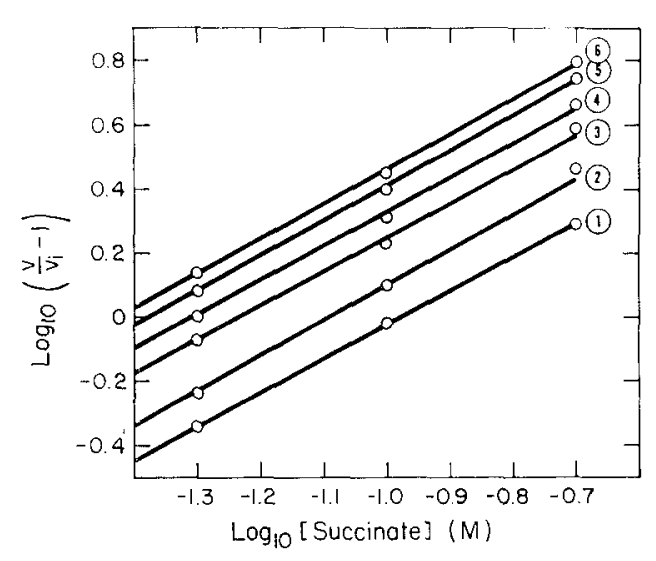

Fig. 5. Determination of the number of succinate ions bound per active site of glycolic acid oxidase. The symbols $v$ and $v_{i}$ refer to the velocity measured in the absence and in the presence of inhibitor, respectively. Lines $\mathrm{I}-6$ wre calculated from rlata obtained at the following glycolate concentrations: $\mathrm{H} / 2500, \mathrm{M} / 5000, \mathrm{M} / 7500, \mathrm{M} / \mathrm{I} 0000, \mathrm{~N} / \mathrm{I} 5000$, and $\mathrm{M} / 20000$, respectively.

mination of the number of inhibitor molecules bound per active site is illustrated for succinate in Fig. 5. The plots for six different glycolate concentrations are essentially parallel, the slopes varying between I.05 and I. Io. Similar values were obtained for oxalate $(n=0.98-1.04)$ and malonate $(n=$ I.03-I.IO). These results show that the complex contains one mole of dicarboxylic acid per active site.

The competitive inhibition of dicarboxylic acids described so far has been for reactions in which glycolate was the substrate. When glyoxalate is the substrate, oxalate and malonate are non-competitive inhibitors. Results obtained with malonate are shown in lig. 6. 


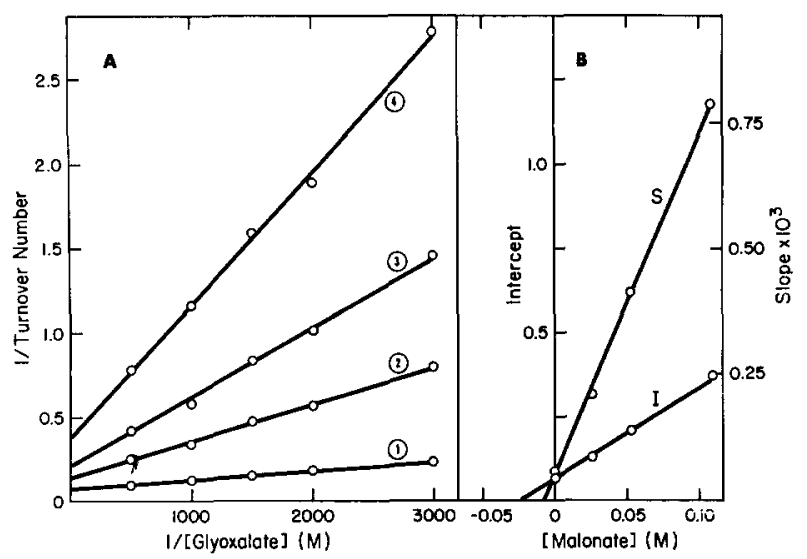

Fig. 6. Inhibition of glyoxalate oxidation by malonate. A. Plot of reciprocal turnover number versus reciprocal glyoxalate concentration. Line $\mathrm{I}$, no inhibitor; Line $2,2.67 \cdot 10^{-2} \mathrm{M}$ malonate; Line 3 , $5.34^{\cdot} \mathrm{IO}^{-2} \mathrm{M}$ malonate; Line $4, \mathrm{I} . \mathrm{I} \cdot \mathrm{IO}^{-1} \mathrm{M}$ malonate. B. Plot of slopes (S) and intercepts (I) versus malonate concentration.

Table II summarizes the inhibition constants for dicarboxylic acids with either glycolate or glyoxalate as substrate. There is good agreement between the values for $K_{i \text { (slope) }}$ determined with either substrate. As for the monocarboxylic acids, $K_{i \text { (slope) }}$ is smaller than $K_{i \text { (intercept) }}$ when glyoxalate is the substrate. A possible explanation for the inhibition patterns observed with the monocarboxylic and dicarboxylic acids will be considered in DIscussion.

\section{Effect of phosphate and arsenate}

The effect of phosphate on enzyme activity with DCIP as the electron acceptor is shown in Fig. 7. Phosphate causes an activation of glycolic acid oxidase. The activation occurs over the same range of phosphate concentrations with either glycolate

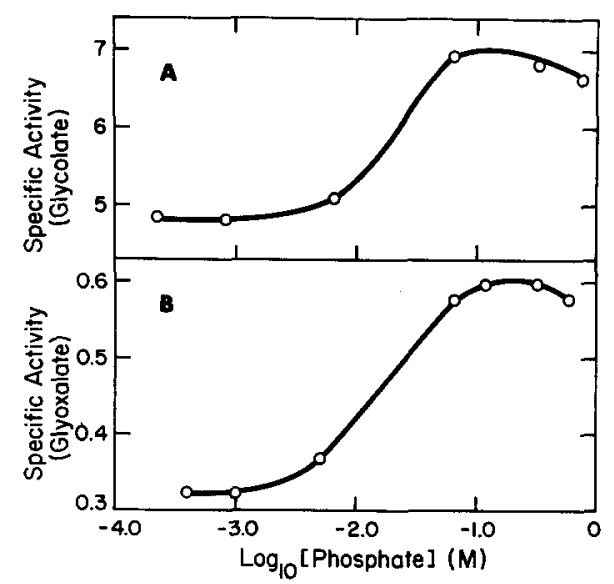

Fig. 7. Phosphate activation in the DCIP assay. A. Specific activity as a function of $\log _{10}$ phosphate concentration (M) with glycolate as substrate. All assays are performed at a glycolate concentration of $6.7 \cdot$ IO $^{-4} \mathrm{M}$ in the standard DCIP assay ${ }^{1}$ except that the phosphate concentration is varied. B. Same as in A, except that glycolate is replaced by $3 \cdot 3 \cdot 10^{-3} \mathrm{M}$ glyoxalate. 
or glyoxalate as substrate. Activity increases from a low value in $5^{\cdot} \mathrm{I}^{-3} \mathrm{M}$ phosphate and reaches an upper level in o.I M phosphate. Decreasing the phosphate concentration below $5^{\cdot 10^{-3}} \mathrm{M}$, or increasing the phosphate concentration above o. I $\mathrm{M}$ does not affect the activity.

A similar activation by phosphate is observed when $\mathrm{K}_{3} \mathrm{~F}+\mathrm{e}(\mathrm{CN})_{6}$ is used as the electron acceptor (Fig. 8). With $\mathrm{K}_{3} \mathrm{Fe}(\mathrm{CN})_{6}$, however, phosphate concentrations above o. $\mathrm{M}$ inhibit the reaction. This inhibition is observed with either glycolate or glyoxalate as substrate. Raising the salt concentration using other anions, such as (1or Hepes, does not cause activation of glycolic acid oxidase. As seen in Fig. 8, similar activities are observed at low concentrations of either Hepes or plospliate. However,

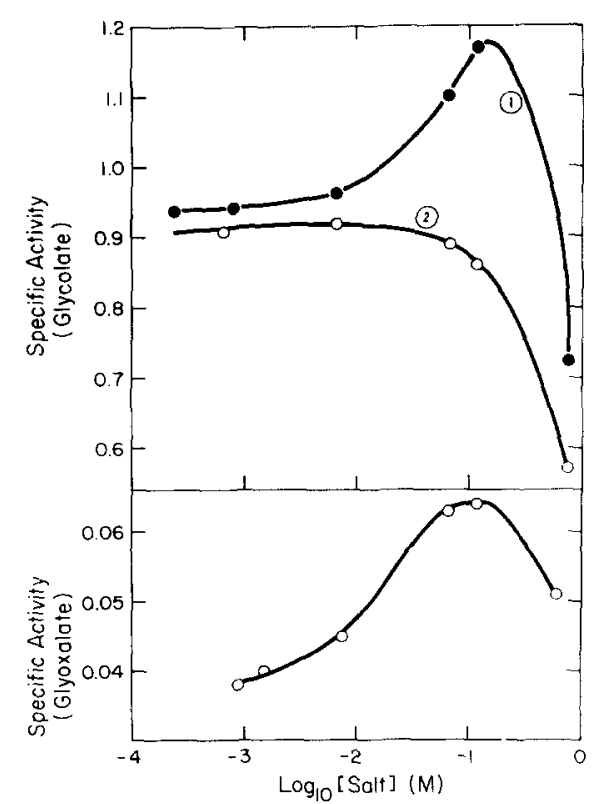

lig. 8. Effect of salt concentration on the $\mathrm{K}_{3} \mathrm{Fe}(\mathrm{CN})_{\mathrm{f}}$ assav. A. Curve 1 , specific activity ats it function of $\log _{10}$ phosphate concentration (M) with glycolate as substrate. All assays are performed at a glycolate concentration of $6.7 \cdot 10^{-4} \mathrm{M}$ in the standard $\mathrm{K}_{3} \mathrm{Fe}(\mathrm{CN})_{6}$ assay ${ }^{1}$ except that the phosphate concentration is varied; Curve 2, same conditions as Curve I except that the salt concentration is varied using Hepes (pH 7.0) instead of phosphate. B. Same conditions as (urve I in A except that glycolate is replaced by $3 \cdot 3^{-10^{-3}} \mathrm{M}$ glyoxalate.

the activity remains constant over a wide range of Hepes concentrations and then decreases at high salt concentrations.

Activation is also observed with arsenate, an anion closely related to phosphate. The activation occurs within the same range of salt concentrations as does phosphate activation and is quantitatively similar to that observed with phosphate. At high concentrations of arsenate, the reaction with $\mathrm{K}_{3} \mathrm{Fe}(\mathrm{CN})_{6}$ as electron acceptor is inhibited.

Activation by phosphate occurs at a salt concentration similar to that required to give appreciable formation of a complex between enzyme and phosphate. Such a complex has been detected spectrophotometrically ${ }^{1}$. Other monovalent and divalent 
anions, which complex with glycolic acid oxidase, do not cause activation, instead they inhibit. All of the inhibition studies described previously for the monocarboxylic and dicarboxylic acids were done at phosphate concentrations which give maximal activity.

\section{DISCUSSION}

This work has shown that in the oxidation of glycolate, coupled with the reduction of DCIP, glycolic acid oxidase shows converging-line kinetics. DICKINsON ${ }^{9}$ and DiCKInSON AND MASSEY ${ }^{10}$ have reported previously that when $\mathrm{O}_{2}$ is the electron acceptor, the enzyme shows parallel-line kinetics. Parallel-line kinetics usually indicate that an enzymic mechanism involves only binary complexes, while convergingline kinetics requires a mechanism that involves a ternary complex ${ }^{\mathbf{1 4}}$. At first sight, it appears that the mechanism of glycolic acid oxidase depends on the electron acceptor. However, this is not necessarily the case. It can be shown ${ }^{9}$ that for a mechanism which involves only binary complexes, the slope of the secondary plot of the intercepts on the $y$-axis of a Lineweaver-Burk plot versus the reciprocal of the concentration of the secondary substrate $\left(i . e . \mathrm{O}_{2}\right)$, should be independent of the nature of the primary substrate (i.e. hydroxy acid). This is not the case with glycolic acid oxidase. DIckINson $^{9}$ found that the slope of such a secondary plot was different for glycolate and glyoxylate. In addition, it is known that under certain limiting conditions, a mechanism involving ternary complexes can give a set of apparently parallel lines in a Lineweaver-Burk plot ${ }^{15}$. Thus it is possible that in glycolic acid oxidase, the mechanism with $\mathrm{O}_{2}$ as electron acceptor is the same as the mechanism with DCIP as acceptor.

Inhibition studies have provided further information on the active site of glycolic acid oxidase. It is proposed that the binding affinity of the monocarboxylic acids reflects both electrostatic and hydrophobic forces. Electrostatic interaction occurs between the negative carboxylate group and a positively charged group at the active site. Evidence for such a charged group was also obtained from studies on the effects of anions on the visible absorption spectrum ${ }^{1}$. The direct correlation found between the binding affinity of a monocarboxylic acid, and the size of its alkyl residue suggests that there is a hydrophobic region near the active site. This region is large enough to accomodate an alkyl residue of at least five carbon atoms without steric interference. A hydrophobic region near the flavin moiety is also suggested by the resolved spectrum observed when anions bind to the enzyme ${ }^{1}$.

One component of the inhibition observed with both monocarboxylic and dicarboxylic acids is due to a direct competition of the inhibitor and the hydroxy acid substrate for oxidized enzyme. This competition may be for the positively charged groups shown to be important in the binding of anions to the protein. Studies on the inhibition by oxalate have suggested that this dicarboxylic acid forms a sterically favorable bidentate complex with two adjacent positively charged groups at the active site. This model for the binding of oxalate can be adapted to explain the binding of $\alpha$-hydroxy acids (see Part C of Fig. 4). In this modified model, one positively charged group interacts with the negative carboxylate group of the substrate. A second positively charged group interacts with the partial negative charge on the oxygen atom of the hydroxyl group. It is necessary to propose this second interaction because electrostatic interaction involving only the carboxylate group cannot 
account for the affinity of the enzyme for a substrate such as glycolate. The free energy change associated with the electrostatic interaction of a single carboxylate group (- I200 cal/mole) corresponds to a dissociation constant of $0.13 \mathrm{M}$. Although the $K_{\mathrm{d}}$ for the enzyme-glycolate complex is not known accurately, preliminary stopped-fiow studies indicate that it is similar in magnitude to $K_{m}\left(K_{m}+.2 \cdot\right.$ Io $M)$. The structure proposed for the enzyme-substrate complex would perhaps also explain the specificity of the enzyme for hydroxy acids substituted at the a position. $A$ considerably decreased binding affinity, analogous to that observed with dicarboxylic acids, is expected for $\beta$-hydroxy acids. Although maximal activity is observed with glycolate as substrate, this enzyme is also active with a number of $\alpha$ hydroxy acids of medium chain length $h^{9,10}$. It is reasonable to assume that hydrophobic forces are especially important in the binding of substrates such as a-hydroxyisocaproate.

Certain aspects of the inhibition studies require further consideration. Why is the inhibition pattern observed with the monocarboxylic acids independent of the nature of the hydroxy acid used as substrate, whereas different inhibition patterins are observed with the dicarboxylic acids when glycolate and glyoxylate are used as substrates? The inhibition of the enzyme by oxalate is also dependent on the nature of the hydroxy acid substrate when $\mathrm{O}_{2}$ is used as the electron acceptor. However, in this case the inhibition is competitive with respect to glyoxylate and non-competitive with respect to glycolate?. All of these results are summarized in Table III.

\section{TABLE III}

SUMMARY OF INHIBITION PATTERNS WITH CARBOXYLIC ACIDS

In all cases the type of inhibition refers to inhibition with respect to the $a$-hydroxy acid substrate. Monocarboxylic acids for System I include all members of the homologous straight-chain serics from acetate to heptanoate. In System 2 acetate, propionate and valerate were tested; no data are available for Systems 3 and 4 . Dicarboxylic acids tested in System $\mathbf{I}$ include oxalate. malonate, and succinate. Oxalate and malonate were tested in System 2. Systems 3 and + refer to clata (taken from ref. 9) obtained only with oxalate.

\begin{tabular}{lll}
\hline System & $\begin{array}{l}\text { Monocarboxylic } \\
\text { acids }\end{array}$ & $\begin{array}{l}\text { Dicarboxylic } \\
\text { acids }\end{array}$ \\
\hline I. Glycolate-DCIP & Non-competitive & Competitive \\
2. Glyoxalate-DCIP & Non-competitive & $\begin{array}{l}\text { Non-competitive } \\
\text { 3. Glycolate }-\mathrm{O}_{2}\end{array}$ \\
4. Glyoxalate-O & & $\begin{array}{l}\text { Non-competitive } \\
\text { Competitive }\end{array}$
\end{tabular}

It is important to emphasize the kinetic differences between non-competitive and competitive inhibition. In competitive inhibition the inhibitor affects only the slope of a Lineweaver-Burk plot. A single inhibition constant, $K_{i \text { (slope), is obtained. }}$ In non-competitive inhibition the inhibitor affects both the slope and the intercept of a Lineweaver-Burk plot. Two inhibition constants, $K_{i \text { (slope) }}$ and $K_{i \text { (intercept), are }}$ obtained. It can be seen from Table III that the inhibitors always affect the slope term. However, whether the inhibitor also affects the intercept term depends on the nature of the hydroxy acid and the electron acceptor.

A possible explanation for the inhibition patterns observed with DCIP as the 
electron acceptor will be considered. A similar argument can also be applied to the $\mathrm{O}_{2}$ system. The converging-line kinetics observed with DCIP as the electron acceptor suggest a mechanism involving a ternary complex. Various mechanisms can be written which will satisfy this requirement. One such mechanism, indicated below, has been arbitrarily selected for the purpose of this discussion. However, as will be seen, the explanation offered to account for the observed inhibition patterns can be applied to other mechanisms.

$$
\begin{gathered}
E+A \underset{k_{2}}{\stackrel{k_{1}}{\rightleftarrows}} E A \underset{k_{4}}{\stackrel{k_{3}}{\rightleftarrows}} E \mathrm{H}_{2} P \\
E \mathrm{H}_{2} P+B \underset{k_{6}}{\stackrel{k_{5}}{\rightleftarrows}} E \mathrm{H}_{2} P B \underset{k_{8}}{\stackrel{k_{7}}{\rightleftarrows}} E P Q \\
E P Q \underset{k_{10}}{\stackrel{k_{9}}{\rightleftarrows}} E Q+P \underset{k_{12}}{\stackrel{k_{11}}{\rightleftarrows} E}+Q
\end{gathered}
$$

where $E$ and $E \mathrm{H}_{2}$ are oxidized and reduced enzyme, $A$ and $B$ are substrates, and $P$ and $Q$ are products.

In this discussion the following assumptions have been made: (I) Dead end inhibition is assumed in all cases, i.e. enzyme-inhibitor complexes are totally inactive. (2) Similar mechanisms are assumed for the oxidation of glycolate and glyoxylate with DCIP as the electron acceptor. Identical or analogous intermediates are formed during the oxidation of either substrate. (3) An inhibitor molecule and a hydroxy acid substrate $(A)$ or its keto acid product $(P)$ cannot bind simultaneously to oxidized enzyme. This is a reasonable assumption since it is known from spectral studies that two anions (i.e. heptanoate and oxalate) cannot be bound simultaneously to oxidized enzyme ${ }^{1}$. Since similar information concerning reduced enzyme is unavailable, this restriction need not hold for the binding of an inhibitor molecule to reduced forms of the enzyme containing $P$.

According to the rules outlined by $C_{L E L A N D}{ }^{16}$, a dead end inhibitor can affect the slope of a Lineweaver-Burk plot if it combines with the same form of the enzyme as the variable substrate $(E$, in the case of substrate $A$ ). If an inhibitor combines with a form of the enzyme which does not bind the variable substrate, it will affect the intercept if the two enzyme forms are in reversible equilibrium. However, it will only affect the slope if the variable substrate adds at some point further along in the sequence than the inhibitor. Since the variable substrate $(A)$ under consideration adds at the start of the sequence, the slope will be affected only if the inhibitor combines with $E$. This will be true for mechanisms which involve any number of additional intermediates or even an entirely different mechanism, provided that $A$ still binds at the start of the sequence. Combination of the inhibitor with any other enzyme form can only affect the intercept.

Enzyme forms incapable of unimolecular reaction with liberation of a substrate or product or isomerization into such a form are called stable enzyme forms in the CLELAND ${ }^{17}$ nomenclature system. According to this nomenclature, $E$ is a stable enzyme form. Enzyme forms capable of unimolecular reaction with the liberation of a substrate or product or isomerization into such a form are called transitory com- 
plexes. For example, $\mathrm{EH}_{2} P$ and $E Q$ are transitory complexes. If an inhibitor combines with a stable enzyme form, the observed $K_{i}$ will be a true $K_{i}$ (and therefore a true $K_{\mathrm{d}}$ ). However, if the inhibitor combines with a transitory complex, the observed $K_{i}$ will be an apparent $K_{i}$. It will be a function of the true $K_{i}$ and certain rate constants in the mechanism ${ }^{11}$.

As discussed above, the slope of a Lineweaver-Burk plot with $A$ as the variable substrate will be affected only if the inhibitor combines with $E$. Since $E$ is a stable enzyme form, the observed $K_{i \text { (slope) }}$ will be a true $K_{i}$ and should be independent of the nature of the substrate $A$. This prediction is satisfied reasonably well by the data presented in Tables I and II. The values obtained for $K_{i \text { (slope) }}$ for the monocarboxylic and dicarboxylic acids with two different substrates are similar.

The intercept of a Lineweaver-Burk plot with $A$ as the variable substrate will be affected if the inhibitor combines with an enzyme form other than $E$. Since all other enzyme forms in the mechanism under consideration are transitory, the $K_{i \text { (intercept) }}$ will not be a true $K_{i}$. The observed $K_{i \text { (intercent) }}$ will be a function of a true $K_{i}$ and certain rate constants in the mechanism and therefore need not be the same for different substrates. In addition, if the inhibitor combines with an intermediate such as $E \mathrm{H}_{2} P$, the true $K_{i}$ need not be the same with different substrates since the nature of $P$ will depend on the nature of $A$. It is therefore possible to define an observed $K_{i \text { (intercept) }}\left(K_{0}\right)$ as a function of a true $K_{i}\left(K_{t}\right)$ and certain rate constants in the enzyme mechanism $(J)$. The values for $K_{\mathrm{t}}$ and $J$ may vary for a given inhibitor depending on the nature of the substrate. The following equations may be written:

$$
\begin{aligned}
& \left(K_{0}^{\mathrm{m}}\right)_{1}=J_{1}^{\mathrm{m}}\left(K_{\mathrm{t}}^{\mathrm{m}}\right)_{1} \\
& \left(K_{0}^{\mathrm{m}}\right)_{2}=J_{2}^{\mathrm{m}}\left(K_{\mathrm{e}}^{\mathrm{m}}\right)_{2} \\
& \left(K_{0}^{\mathrm{d}}\right)_{1}=J_{1}^{\mathrm{d}}\left(K_{\mathrm{t}}^{\mathrm{d}}\right) \\
& \left(K_{0}^{\mathrm{d}}\right)_{2}=J_{2}^{\mathrm{d}}\left(K_{\mathrm{t}_{2}}^{\mathrm{d}}\right)_{2}
\end{aligned}
$$

The superscripts $m$ and $d$ refer to the monocarboxylic and dicarboxylic acid series, respectively. The numbers $\mathrm{I}$ and 2 refer to glycolate and glyoxylate, respectively. It will be noted that Eqn. 9 defines an inhibition constant not detected kinetically since dicarboxylic acids only affect the slope of a Lineweaver-Burk plot when glycolate is the variable substrate. Kinetically, $\left(K_{0} \mathrm{~d}\right)_{1}$ is infinitely large. Since finite values are obtained for $\left(K_{0} \mathrm{~d}\right)_{2}$ (i.e. dicarboxylic acids affect the intercept of a Lineweaver-Burk plot with glyoxylate as the variable substrate), the inequality expressed in Eqn. I I will be true.

$$
J_{1}^{\mathrm{d}}\left(K_{\mathrm{t}}^{\mathrm{d}}\right)_{1} \gg J_{2}^{\mathrm{d}}\left(K_{\mathrm{t}}^{\mathrm{d}}\right)_{2}
$$

The equality expressed in Eqn. $\mathrm{r} 2$ is reasonable since the values obtained for $K_{i \text { (intercept) }}$ in the monocarboxylic acid series are approximately the same with glycolate and glyoxylate as the variable substrates (see Table I). 


$$
J_{1}^{\mathrm{m}}\left(K_{\mathrm{t}}^{\mathrm{m}}\right)_{1}=J_{2}^{\mathrm{m}}\left(K_{\mathrm{t}}^{\mathrm{m}}\right)_{2}
$$

Since similar mechanisms are assumed, enzyme intermediates formed during glycolate and glyoxylate oxidation will either be identical (e.g. EQ) or analogous (e.g. $E \mathrm{H}_{2} P$ ). In general, with two classes of enzyme intermediates and two classes of inhibitors, three combinations of enzyme intermediate-inhibitor complexes are possible. In Case I both monocarboxylic and dicarboxylic acids combine with an intermediate such as $E Q$. For a given substrate, the value for $J$ will depend only on the enzyme intermediate with which the inhibitor combines ${ }^{11}$. In addition, it is clear that the $K_{\mathrm{t}}$ will be independent of the nature of substrate $A$. The following equations can be written:

$$
\begin{aligned}
& J_{1}^{\mathrm{m}}=J_{1}^{\mathrm{d}}=J_{1} \\
& J_{2}^{\mathrm{m}}=J_{2}^{\mathrm{d}}=J_{2} \\
& \left(K_{\mathrm{t}_{1}}^{\mathrm{d}}\right)_{1}=\left(K_{\mathrm{t}^{\mathrm{d}}}^{\mathrm{d}}\right)_{2}=K_{\mathrm{t}}^{\mathrm{d}} \\
& \left(K_{\mathrm{t}}^{\mathrm{m}}\right)_{1}=\left(K_{\mathrm{t}}^{\mathrm{m}}\right)_{2}=K_{\mathrm{t}}^{\mathrm{m}}
\end{aligned}
$$

Eqns. I3-I6 can be substituted into Eqns. II and I2 to give Eqns. I7 and I8.

$$
\begin{aligned}
& J_{1} K_{\mathrm{t}}^{\mathrm{d}} \gg J_{2} K_{\mathrm{t}}^{\mathrm{d}} \\
& J_{1} K_{\mathrm{t}}^{\mathrm{m}}=J_{2} K_{\mathrm{t}}^{\mathrm{m}}
\end{aligned}
$$

However, when Eqn. I7 is divided by Eqn. I8, an impossible inequality is obtained and therefore Case $\mathrm{I}$ is ruled out.

In Case 2 both monocarboxylic and dicarboxylic acids combine with an intermediate such as $E_{2} P$. Eqns. I3 and $\mathrm{I}_{4}$ also apply in this situation but Eqns. I5 and I6 need not apply. Under these conditions Eqn. I9 is obtained.

$$
\frac{\left(K_{\mathrm{t}}^{\mathrm{d}}\right)_{1}}{\left(K_{\mathrm{t}}^{\mathrm{m}}\right)_{1}} \gg \frac{\left(K_{\left.t^{2}\right)_{2}}^{\mathrm{d}}\right.}{\left(K_{\mathrm{t}}^{\mathrm{m}}\right)_{2}}
$$

Eqn. I9 states that the relative affinity of $E \mathrm{H}_{2} P$ for monocarboxylic and dicarboxylic acids varies greatly with the nature of $P$. If this possibility is excluded, Case 2 can be eliminated.

In Case 3 dicarboxylic and monocarboxylic acids combine with different enzyme forms (e.g. $E Q$ and $E \mathrm{H}_{2} P$ ). Let us assume that the true $K_{i}$ is relatively unaffected by the nature of $P$. Eqns. 15 and 16 are assumed to be true. Eqns. 13 and 14 need not apply. Under these conditions Eqn. 20 is obtained. 


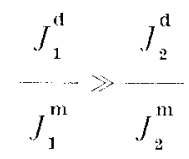

Lsing rules described by CLELAND ${ }^{11}$, it is possible to determine an expression for $J$ in terms of substrate-specific rate constants. The expression for $J$, associated with the formation of a given enzyme intermediate-inhibitor complex is determined by performing the following operations: (I). The distribution equations (i.e. $E / E_{\mathrm{t}}$ ) are calculated according to the method of KING AND ALTMAN ${ }^{18}$ in terms of kinetic constants. (2) The rate equation in the presence of inhibitor is derived by the same method used to derive the equation in the absence of the inhibitor except that the numerator of the distribution equation of the enzyme intermediate with which the inhibitor combines is multiplied by the factor $\left.(I+i I] / K_{t}\right)$. (3) The intercept term in the final rate equation (with $A$ as the variable substrate) is set equal to zero and solved for $I$. When the intercept term is zero $\mid I]=J K_{\mathbf{t}}$. If dicarboxylic acids combine with $E \mathrm{H}_{2} I$ ' and monocarboxylic acids combine with $E Q$, substitution of expressions obtained for $J$ into Eqn. 20 yields Eqn. 2 I.

$$
\left[\frac{k_{5} k_{7} k_{9}}{k_{6} k_{9}+k_{7} k_{9}+k_{6} k_{8}}\right]_{1} \geqslant\left[\frac{k_{5} k_{7} k_{9}}{k_{6} k_{9}+k_{7} k_{9}+k_{6} k_{8}}\right]_{2}
$$

The numbers I and 2 again refer to glycolate and glyoxylate, respectively. It will be noted that the rate constants contained in Eqn. $2 \mathrm{I}$ can vary depending on the nature of the substrate $A$. Since it is known that glycolate and glyoxylate are oxidized at different rates, there is no reason to assume that these rate constants will be identical for the two substrates. This is consistent with the inequality expression.

In Case 3, qualitatively different inhibition patterns can result simply as a function of quantitative differences in the rate constants associated with the oxidation of glycolate and glyoxylate. Let us continue to assume that the true $K_{i}\left(K_{t}\right)$ is relatively unaffected by the nature of $P$ (i.e. Eqns. 15 and 16 are valid). Under these conditions, $J_{1} \mathrm{~m}$ and $J_{2} \mathrm{~m}$ must be similar in magnitude since similar values for $K_{i \text { (intercept) }}$ are observed for monocarboxylic acids with glycolate and glyoxalate as substrates. However, suppose $J_{1}{ }^{\mathrm{d}}$ is 30 times larger than $J_{2}{ }^{\mathrm{d}}$. The $K_{i \text { (intercept) observed }}$ for oxalate with glyoxalate as substrate is $\mathrm{I} .4 \cdot \mathrm{IO}^{-3} \mathrm{M}$. Using Eqns. 9 and $\mathrm{ro}$, a $K_{\text {(intercept) }}$ of approximately $4 \cdot \mathrm{IO}^{-2} \mathrm{M}$ is predicted for oxalate inhibition with glycolate as substrate. This value is roo times larger than the value obtained for $K_{i \text { (slope) }}$ in the case of oxalate inhibition. Under these conditions, the concentration of oxalate required to double the intercept term would increase the slope term by a factor of 100 (see Eqn. I). Kinetically, it would be extremely difficult to detect an effect of the inhibitor on the intercept, and the inhibition would appear to be competitive.

Given the assumptions outlined at the beginning of this discussion, it is possible to rule out Case $\mathrm{r}$. At the present time it is not possible to distinguish between Cases 2 and 3. Fither case could account for the inhibition patterns observed with monocarboxylic and dicarboxylic acids. However, Case 3 is somewhat more attractive since the inhibition patterns can be explained solely in terms of differences 
in rate constants associated with two substrates which are found experimentally to be oxidized at quite different rates.

\section{ACKNOWLEDGMENTS}

This work was supported by Grant GM IIIo6 from the U.S. Public Health Service. M. S. is the recipient of a National Science Foundation Predoctoral Fellowship.

\section{REFERENCES}

I M. Schuman and V. Massey, Biochim. Biophys. Acta, 227 (1971) 500.

2 A. De Kok and C. Veeger, Biochim. Biophys. Acta, 56 (I962) 4 I 3.

3 P. A. Sullivan, in H. Kamin, Flavins and Flavoproteins, University park Press, Baltimore, I970, in the press.

4 D. V. Dervartanian and C. Veeger, Biochim. Biophys. Acta, 92 (1964) 233.

5 P. K. TuBBs, Biochem. $J$., 82 (1962) 36.

6 M. Fonda And B. M. Anderson, J. Biol. Chem., 243 (1968) I93I.

7 S. Dikstein, Biochim. Biophys. Acta, 36 (1959) 397.

8 F. Bergmann and R. Segal, Biochem. J., 58 (I954) 692.

9 F. M. Dickinson, Doctoral dissertation, University of Sheffield, England, 1963.

Io F. M. Dickinson and V. Massey, Biochem. J., 89 (I963) 53 P.

i I W. W. Clenland, Biochim. Biophys. Acta, 67 (I963) I 73 .

I2 W. P. Jencks, Catalysis in Chemistry and Enzymology, McGraw-Hill, New York, I969, pp. $323-436$.

i3 W. Kauzmann, in C. B. Afinsen, Jr., M. L. Anson, K. Bailey and J. T. Edsall, Advances in Protein Chemistry, Academic Press, New York, I959, p. I.

I4 R. AlberTy, in F. F. Nord, Advances in Enzymology, Vol. I7, Interscience, New York, 1956, p. $\mathbf{I}$.

I5 G. Palmer and V. Massey, in T. P. Singer, Biological Oxidations, Interscience, New York, I968, p. 263.

I6 W. W. Cleland, Biochim. Biophys. Acta, 67 (1963) I88.

I 7 W. W. Cleland, Biochim. Biophys. Acta, 67 (1963) ro4.

is E. L. King and C. Altman, J. Phys. Chem., 60 (1956) 1375.

Biochim. Biophys. Acta, 227 (I97I) 521-537 